\title{
Probabilistic Reliability Management Approach and Criteria for Power System Real-time Operation
}

\author{
Efthymios Karangelos and Louis Wehenkel \\ Department of EE\&CS - Institut Montefiore, University of Liège, Belgium \\ \{e.karangelos, 1.wehenkel\}@ulg.ac.be
}

\begin{abstract}
This paper develops a probabilistic approach for power system reliability management in real-time operation where risk is a product of $i$ ) the potential occurrence of contingencies, ii) the possible failure of corrective (i.e., post-contingency) control and, iii) the socio-economic impact of service interruptions to end-users. Stressing the spatiotemporal variability of these factors, we argue for reliability criteria assuring a high enough probability of avoiding service interruptions of severe socio-economic impact by dynamically identifying events of nonnegligible implied risk. We formalise the corresponding decision making problem as a chance-constrained two-stage stochastic programming problem, and study its main features on the single area IEEE RTS-96 system. We also discuss how to leverage this proposal for the construction of a globally coherent reliability management framework for long-term system development, midterm asset management, and short-term operation planning.
\end{abstract}

Index Terms-Power System Operation, Reliability Criteria, Reliability Management, Two-stage Stochastic Programming.

口

\section{INTRODUCTION}

Nowadays, the necessity of revisiting power system reliability management is becoming a matter of consensus. While the utility of probabilistic approaches has long been advocated (see, for instance [1]) the migration from the deterministic N-1 approach is yet to be achieved [2]. Amongst the key barriers, the multi-stage character of the decision making problem poses several complexity challenges [3], [4]. In the present work we focus on the latest decision making opportunity, namely realtime operation [5], as a first step towards the development of a coherent approach across all decision-making horizons.

In real-time operation, the challenge of reliability management is to efficiently mitigate the potential impacts of contingency events by means of preventive and/or corrective control. To this end, it is necessary to jointly take into account: i) probabilities of contingencies, ii) possibilities of corrective control failures, and, iii) service interruption costs to endusers [6]. Notably, the aforementioned factors are variable both across different geographical regions and across different time instants. This spatiotemporal variability calls to question the utility of approaches based on static subsets of credible

The research leading to these results has received funding from the European Union Seventh Framework Programme (FP7/2007-2013) under grant agreement No 608540, project acronym GARPUR. The scientific responsibility rests with the authors. contingencies (such as the N-1 or N-k approaches). Indeed, in the presence of spatiotemporal variability, these can not consistently maintain the system reliability level nor optimise its socio-economic impact on system end-users [7], [8].

\section{A. Proposal}

Motivated by these facts, we propose a probabilistic Reliability Management Approach and Criterion (RMAC) as a synthesis of the three following basic ingredients:

1) A reliability target: it ensures that the probability of reaching unacceptable system states (for instance, instabilities and/or service interruptions of large size, duration, geographical extent) is lower than a fixed tolerance.

2) A socio-economic objective: it prescribes to minimise a socio-economic cost function composed of: i) the costs incurred by the application of preventive and/or corrective (i.e. post-contingency) decisions, and, ii) the aggregated risk of service interruptions implied by these decisions, expressed as the expected value of service interruption costs over the set of considered combinations of contingencies and corrective control failure modes.

3) A discarding principle: it allows to neglect in items 1) and 2) a subset of contingencies only under the condition that the implied residual risk, expressed as the expected value of service interruption costs over that neglected subset, is guaranteed to be below a fixed threshold.

The rest of this paper is organised as follows: section II provides a motivation and formal statement of the proposed RMAC; section III discusses the required efforts in terms of modelling; section IV provides a case study on the single area of the IEEE RTS-96 test system [9]; section V recapitulates, discusses the future directions in this work, and the more general objective to establish a consistent probabilistic approach across all TSO reliability management contexts (i.e. from realtime towards longer term horizons). Supplementary material, see [10], provides detailed mathematical formulations and data sets used in this paper, for the sake of reproducibility.

\section{Probabilistic RELIABILITY MANAGEMENT}

In this section, we introduce the general statement of the real-time operation reliability management problem considered in this paper. On this basis, we develop the proposed probabilistic RMAC and briefly discuss its relation to the currently used N-1 approach. 


\section{A. Preliminaries \& Notation}

We consider a real-time operation horizon over which power injections as well as weather conditions can be regarded as deterministic (known) parameters (say $\Delta t \sim 15^{\prime}-60^{\prime}$ ). In other words, uncertainty relates only to the possible realisation of contingency events (e.g., the unplanned failure or outage of one or more system components) as well as to the effectiveness of the application of post-contingency corrective controls.

Let us denote by $x_{0}$ the current operating state of the system and by $\mathcal{C}$ the set of possible contingency events that may happen within the concerned horizon (including the "no disturbance case"). Reliability management in realtime aims at choosing a joint preventive/corrective control strategy $u \in \mathcal{U}\left(x_{0}\right)$ consisting of a preventive control decision $u_{0} \in \mathcal{U}_{0}\left(x_{0}\right)$ and a set of corrective control decisions $\left\{u_{c} \in \mathcal{U}_{c}\left(x_{0}, u_{0}, c\right), \forall c \in \mathcal{C}\right\}$, a $u_{c}$ being precomputed to be applied after the occurrence of the contingency $c$. We represent the transition to the post-contingency state by $x_{0} \rightarrow x_{c}=f_{0}\left(x_{0}, u_{0}, c\right), \forall c \in \mathcal{C}$.

Concerning the probability of occurrence of contingencies and corrective control behaviors, we focus on the role of the prevailing weather conditions over the area of interest [11]. To this end, we denote by $w_{0}$ the prevailing weather conditions and regard the probability of a contingency $c$ as a function $\pi_{c}\left(w_{0}\right) \in[0,1]$, with $\sum_{c \in \mathcal{C}} \pi_{c}\left(w_{0}\right)=1$. As for the uncertainty on the effectiveness of corrective control, we denote by $\mathcal{B}$ the set of possible corrective control behaviour modes and use symbol $\pi_{b}\left(w_{0}\right){ }^{\prod}$ to represent the probability of realization of any possible behavior $b \in \mathcal{B}$, with $\sum_{b \in \mathcal{B}} \pi_{b}\left(w_{0}\right)=1$. We also represent the transition from a post-contingency state, driven by the (subsequent) application of the respective corrective control $u_{c}$ and the realization of its behavior $b$, by $x_{c} \rightarrow x_{c}^{b}=$ $f_{1}\left(x_{c}, u_{c}, b\right), \forall c, b \in \mathcal{C} \times \mathcal{B}$.

Finally, we shall use functions $C P\left(x_{0}, u_{0}, w_{0}\right)$ and $C C\left(x_{c}, u_{c}, w_{0}\right)$ for the costs of preventive and corrective controls respectively, the latter being incurred conditionally on the realization of any contingency $c \in \mathcal{C}$. As for the socio-economic impact of service interruptions, we assume that these are measurable by a severity function, denoted as $S\left(x_{c}^{b}\left(u_{0}, u_{c}\right), w_{0}\right)$. Notice that we express these three functions in monetary units and explicitly indicate their possible dependance on weather conditions.

In a theoretical context, it can be argued that choosing the joint preventive/corrective control strategy $u \in \mathcal{U}\left(x_{0}\right)$ so as to minimise the compound expectation of the socio-economic costs of reliability management, as in (1), is the approach maximising the welfare of the electricity system stakeholders,

$$
\begin{aligned}
\min _{u \in \mathcal{U}\left(x_{0}\right)} & \left\{C P\left(x_{0}, u_{0}, w_{0}\right)+\sum_{c \in \mathcal{C}} \pi_{c}\left(w_{0}\right) \cdot\left[C C\left(x_{c}, u_{c}, w_{0}\right)\right.\right. \\
& \left.\left.+\sum_{b \in \mathcal{B}} \pi_{b}\left(w_{0}\right) \cdot S\left(x_{c}^{b}\left(u_{0}, u_{c}\right), w_{0}\right)\right]\right\} .
\end{aligned}
$$

${ }^{1}$ For ease of notation, we leave the possible dependence of $\pi_{b}$ on $x_{c}=$ $f_{0}\left(x_{0}, u_{0}, c\right)$ and $u_{c}$ implicit.
In practice, the usefulness of such a risk neutral approach, providing no assurance on the avoidance of extremely detrimental service interruptions appears questionable. We note that, by such an approach, the level of service provided by the system remains sensitive to volatile economic variables, such as the preventive control costs. In parallel, an accurate model of the severity function needed to translate service interruptions into a monetary value may not be available for all possible service interruption situations.

In addition, modelling in the time domain the dynamic trajectories of the system state induced by the chosen decision and the potential realization of contingencies and corrective control behaviours can be very complex. Last but not least, we must notice that the space $\mathcal{C} \times \mathcal{B}$ of all possible combinations of contingencies and corrective control behaviours can be too large, making the optimisation of (1) not tractable.

\section{B. Proposed reliability management approach and criterion}

Reliability target. Let us denote by $\left(x_{0}, x_{c}, x_{c}^{b}\right)$ a system trajectory and by $X_{a}$ a set of acceptable trajectories, i.e. implying an acceptable level of service to the system endusers. We start by formalising a reliability target of avoiding, with certain confidence, the realisation of unacceptable trajectories. In other words, we seek to ensure that the sum of the probabilities of contingencies and respective corrective control behaviours leading to unacceptable trajectories is smaller than a fixed tolerance, and express this as,

$\mathbb{P}\left\{\left(x_{0}, x_{c}, x_{c}^{b}\right) \in X_{a} \mid(c, b) \in \mathcal{C} \times \mathcal{B}\right\} \geq\left(1-\varepsilon_{R T}\right)$,

where $\varepsilon_{R T} \in[0 ; 1]$ denotes the fixed tolerance.

Notice that the set $X_{a}$ as well as the tolerance level $\varepsilon_{R T}$ serve as meta-parameters of the proposed RMAC. The set $X_{a}$ may be expressed in the form of the classical constraints of a security constrained optimal power flow formulation, corresponding to the relevant operational limits of the system in question. The tolerance level $\varepsilon_{R T}$ allows to abide the realization of unacceptable trajectories provided that the respective probability remains sufficiently low. Notice that the value of $\varepsilon_{R T}=0$, i.e. the most stringent target, may preclude the choice of corrective actions with non-zero failure probability. This is because the possible failure of such actions normally implies an unacceptable system state. Evidently, the value of $\varepsilon_{R T}=1$ renders the reliability target ineffectual.

Discarding principle. For a subset $\mathcal{C}_{c} \subset \mathcal{C}$ and a reliability management strategy $u \in \mathcal{U}\left(x_{0}\right)$, we define the residual risk associated to the contingencies $c \in \mathcal{C} \backslash \mathcal{C}_{c}$ by

$R_{\mathcal{C} \backslash \mathcal{C}_{c}}(u)=\sum_{c \in \mathcal{C} \backslash \mathcal{C}_{c}} \pi_{c}\left(w_{0}\right) \sum_{b \in \mathcal{B}} \pi_{b}\left(w_{0}\right) S\left(x_{c}^{b}\left(u_{0}, u_{c}\right), w_{0}\right)$.

For any $\mathcal{C}_{c} \subset \mathcal{C}$ and strategy $u \in \mathcal{U}\left(x_{0}\right)$, this residual risk measures the approximation error of the cost function (1) when using only the subset $\mathcal{C}_{c}$ to compute the expected severity. Hence, if this latter quantity can be ascertained to be small enough for any relevant reliability management strategy, given the precision that can be reasonably targeted for the evaluation 
of (1), it means that one can reasonably neglect the elements of $\mathcal{C} \backslash \mathcal{C}_{c}$ in the RMAC formulation.

In practice, among the many possible contingencies, a large number have a very small probability and/or lead to negligible service interruptions. On the other hand, the accuracy of the computation of (1) is intrinsically bounded by the quality of the available models of probabilities and service interruptions. It therefore is not necessary to formulate an RMAC which would explicitly cover all possible contingencies. On this premise, we allow to discard from (1), (2) a subset of contingency events $\mathcal{C} \backslash \mathcal{C}_{c}$ provided that the implied residual risk satisfies the following constraint:

$R_{\mathcal{C} \backslash \mathcal{C}_{c}}(u) \leq \Delta E_{R T}$

Here, we display the accuracy threshold $\Delta E_{R T}$ as the third meta-parameter of the proposed RMAC and notice that larger values of $\Delta E_{R T}$ should allow for identifying optimal strategies with respect to smaller subsets $\mathcal{C}_{c}$, in essence reducing the computational complexity of the problem in question at the expense of adopting greater residual risk. We should also underline that, under the mild assumption that the severity function is bounded from above, a conservative upper bound on the residual risk value can be employed to reduce (4) to a bound on the joint probability of "discarded" contingencies $c \in \mathcal{C} \backslash \mathcal{C}_{c}$. Indeed, denoting this upper bound by $S^{\max }$, we can re-state (4) as in (5).

$\sum_{c \in \mathcal{C} \backslash \mathcal{C}_{c}} \pi_{c}\left(w_{0}\right) \leq \Delta E_{R T} / S^{\max }$.

\section{RMAC statement}

Synthesizing the socio-economic cost minimization objective of (1) with the reliability target of (2) and the discarding principle of (4), we state the proposed RMAC for real-time operation as in (6) - (8).

$$
\begin{aligned}
\min _{u \in \mathcal{U}\left(x_{0}\right)} & \left\{C P\left(x_{0}, u_{0}, w_{0}\right)+\sum_{c \in \mathcal{C}_{c}} \pi_{c}\left(w_{0}\right) \cdot\left[C C\left(x_{c}, u_{c}, w_{0}\right)\right.\right. \\
& \left.\left.+\sum_{b \in \mathcal{B}} \pi_{b}\left(w_{0}\right) \cdot S\left(x_{c}^{b}\left(u_{0}, u_{c}\right), w_{0}\right)\right]\right\} \\
\text { s.t. } & \mathbb{P}\left\{\left(x_{0}, x_{c}, x_{c}^{b}\right) \in X_{a} \mid(c, b) \in \mathcal{C}_{c} \times \mathcal{B}\right\} \geq\left(1-\varepsilon_{R T}\right)
\end{aligned}
$$

while

$$
R_{\mathcal{C} \backslash \mathcal{C}_{c}}(u) \leq \Delta E_{R T}
$$

Notice that we highlight condition (8) by using the "while" statement to point to the fact that, in practice, one may break the reliability management process in the steps of iteratively i.) choosing $\mathcal{C}_{c}$, ii.) then solving (6.7), and iii.) checking (8) and if not satisfied, expanding $\mathcal{C}_{c}$. Such practice may well exploit the reduced complexity of evaluating (8) for a given strategy $u \in \mathcal{U}\left(x_{0}\right)$ with respect to the complexity of the optimization problem (6-7). Nevertheless, in principle (8) can be seen as just another constraint imposed on the optimisation problem.

\section{Discussion: the proposed RMAC vs " $N-1$ "}

The proposed RMAC results from the combination of the socio-economic optimisation objective function (6) with a reliability target (7), in the form of a chance constraint avoiding undesirable contingency responses, and a discarding principle (8), stating the sought level of accuracy in the monetary evaluation of contingency responses.

Beyond the physical models (in the form of the transition functions $f_{0}$ and $f_{1}$ ) and the economic model (in the form of the cost functions $C P, C C$ and severity $S$ ), this RMAC is based on three additional meta-parameters, namely $X_{a}, \varepsilon_{R T}$ and $\Delta E_{R T}$, which allow one to tune its risk-averseness with respect to a pure minimisation of socio-economic costs, while taking into account limitations in computational resources and in the accuracy of the used physical and economic models.

In the light of our criterion, which allows to dynamically adjust the set of considered contingencies, while taking into account both weather conditions and the possibility of corrective control failure, the classical "N-1" approach consists of using an a priori fixed set $\mathcal{C}_{N-1}$ irrespectively of weather conditions while implicitly assuming that (4) holds true, a tolerance level $\varepsilon_{R T}=0$, and only envisages a single possible behaviour of corrective control (no failure).

\section{MODELLING REQUIREMENTS \& IMPLEMENTATION}

In this section we first briefly discuss the general modelling requirements of the proposed RMAC, and then describe in more detail the mathematical model that we have implemented and used in our case studies.

\section{A. General modelling requirements}

In order to exploit the proposed probabilistic RMAC in practice, we need to address several additional modelling issues normally not tackled in current practice. Indeed, the value of the approach lies in its ability to dynamically identify and then cover a subset of contingencies $\mathcal{C}_{c}$ based on the estimation of the residual risk and the probability of violating the chance constraint on the acceptable system trajectories. Both these constraints call for modelling the spatio-temporal variation of the following items:

- Probabilities of contingencies $\pi_{c}\left(w_{0}\right)$ as a function of weather conditions mainly, but in principle also dependent on component loading conditions.

- Failure modes $\mathcal{B}$ of corrective controls and their respective probabilities $\pi_{b}$, in principle as well function of weather conditions and (possibly to a lesser extent) on component loading status.

- Physical contingency responses $f_{0}$ and $f_{1}$, in particular under corrective control failure assumptions, and their socio-economic consequences on the system end-users in the form of a severity function; the latter are often strongly dependent on weather conditions.

In addition to these modelling items necessary to enable the checking of constraints (7) and (8) of the proposed RMAC, obviously the modelling of preventive and corrective control 


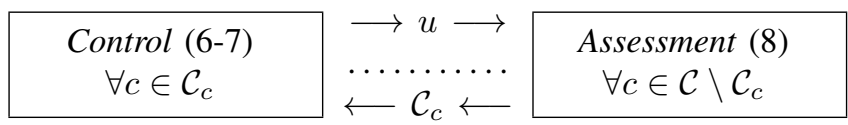

Figure 1. Iterative solution approach

costs are required to rank strategies in terms of their socioeconomic impact, as expressed by the objective (6).

\section{B. Mathematical model used in the implementation}

As a first step towards the development of tractable and scalable approximations to the problem in question, we decompose in this work the RMAC statement of (6 8) by treating the problem of identifying an optimal preventive/corrective strategy (6,7) and the discarding principle (8) in an iterative manner (see Figure 11). More specifically, we begin by seeking a preventive/corrective strategy under a given subset of contingencies $\mathcal{C}_{c} \subset \mathcal{C}$ as per (6.7), using for instance the subset of single outages $\mathcal{C}_{N-1}$ as a starting point in the iterative process. Subsequently we evaluate $(8)$ to identify whether the subset under consideration can be considered as an acceptable approximation of $\mathcal{C}$. If this is not the case, we exploit the findings of the assessment of the residual risk implied by $\mathcal{C} \backslash \mathcal{C}_{c}$ to form a new subset and return to the solution of (6.77).

Concerning the decision making problem of (6,7) we adopt the following approximations, with the sole purpose of facilitating the clear presentation of the features of the proposed approach:

- We define the initial power injection and demand as the result of a perfectly competitive market for electricity with no transmission constraints.

- We employ the DC power flow approximation [12]. In this context, we consider the re-dispatch of the power output of any generating unit to form the set of available preventive actions. Following the occurrence of any contingency, we consider the adjustment of phase shifting transformers in addition to generation re-dispatch as available corrective actions. Regarding generating units, we consider both the minimum/maximum power output restrictions as well as ramping restrictions between any successive dispatch stage. As for the adjustments of phase shifting transformers, we consider the respective minimum and maximum limits.

- We model the set of acceptable system trajectories $X_{a}$ by means of a set of constraints expressing the system operational limits in the pre- and post-contingency states. We underline that, in our implementation, an acceptable post-contingency trajectory concerns the achievement of an equilibrium without any loss of load both i) in the short-term following the occurrence of any contingency and prior to the realization of the behavior of corrective control, and, ii) following the realization of corrective control behavior. We employ short-term thermal ratings to express the limits on the power flowing through the network components in the former case.
- In the event of any violation of the system operational limits, i.e. under any unacceptable trajectory, we employ a pessimistic approximation of the implied severity level. In particular, we consider that any violation of the system operational limits implies the realization of the upper bound of the severity value $S^{\max }$. We compute such value by multiplying the end-user demand with the (spatially and temporally variable) value of lost load (voll) for the time duration of interest. Notice that we adopt this pessimistic approach in the interest of conservativeness, and particularly to avoid any potential under-estimation of the severity levels induced by other approximations in our model.

- To model the possible influence of prevailing weather conditions on the probability of any contingency event, we start from the fundamental model of [11], linking component forced outage rates to a discretized set of weather conditions. Accordingly, we derive contingency probabilities from such weather related forced outage rates as per [13].

- Regarding the failure of corrective control, we begin by assuming that any elementary control operation (e.g. the re-dispatch of any single generating unit, the adjustment of any single phase shifting transformer, etc.) may either work or fail with a known probability. On this basis, we approximate the probability of failure of any chosen $\left\{u_{c} \in \mathcal{U}_{c}\left(x_{0}, u_{0}, c\right), \forall c \in \mathcal{C}\right\}$ as the minimum between the sum of the failure probabilities of all concerned elementary control operations and the value of one.

- We approximate the first term of objective function (6) as the summation, for all generating units, of a linear cost function of the re-dispatch quantity with respect to the initial dispatch (i.e. market clearing outcome). We also approximate the second term, i.e. the cost of corrective control, by summing up for all generating units the maximum between zero and a linear cost function of the re-dispatch with respect to the pre-contingency operating point. Notice that we account for this cost irrespectively of the possible realization of the failing behavior of corrective control.

The above lead to a mixed-integer linear programming (MILP) formulation of (6) - (7), which is presented in full as supplementary material at [10].

In the second step of our solution approach, and particularly the computation of the residual risk for the evaluation of (8), we have implemented the cascading failure simulator presented in [14], which is a modified version of the model originally introduced in [15]. Referring the interested reader to [14], [15] for a detailed presentation of this simulation model, we should briefly introduce that this DC power flow based algorithm starts from any triggering event and involves the sequential simulation of relay-based trippings of overloaded branches, generator trippings and load shedding events, while taking into account the accumulated overloading of any branch over time to define the temporal characteristics of the cascade 
evolution process.

Notice that we indeed exploit the reduced complexity of the assessment problem to perform the described quasi-static simulation of the possible occurrence of contingencies $c \in \mathcal{C} \backslash$ $C_{c}$, while in the optimization problem of (6.7) we resort to the conservative, yet less complex, approximation of the implied consequences of violating the system operational limits.

\section{Demonstrative Case Studies}

In this section, we demonstrate the key features of the proposed RMAC through a set of case studies based on the well-known IEEE RTS [9]. We shall consider 2 distinctive cases, namely case $A$ and case $B$, corresponding to the 1-hour interval $[12: 00 ; 13: 00)$ on the Monday of week 23 (summer) and week 46 (winter) respectively. We shall also consider an annual peak load of $3000 \mathrm{MW}$, resulting to a total load of $2509 \mathrm{MW}$ in case $A$ and $2536 \mathrm{MW}$ in case $B$.

With the purpose of demonstrating the proposed approach, we consider that the set $\mathcal{C}$ (i.e. the set of mutually exclusive and collectively exhaustive contingency events) consists of the forced outage of any single transmission component and the common mode forced outage of branches [(A12-1,A13-1);(A18,A20);(A25-1,A25-2);(A30,A34);(A311,A31-2);(A32-1,A32-2);(A33-1,A33-2)], in addition to the pseudo-contingency of no disturbance. In oder to examine the adaptability of the proposed RMAC to the temporal variability of contingency probabilities, in case $A$ we use the permanent forced outage rates listed in [9] to compute the probability of any contingency while in case $B$ we arbitrarily assume that due to adverse weather conditions forced outage rates are increased by $10 \%$, with the exception of the cables [A1;A10].

Similarly, we use the voll data found in [16] for case $A$ and assume that in case $B$ the voll at each node is increased by $(15 \%)$ in order to also investigate the adaptability of the proposed RMAC to the variability of this factor as well. In both cases, we use the generation cost data described in [17], to establish the generation dispatch at the start of the real-time interval in question through the clearing of the perfectly competitive market with no transmission constraints. To compute re-dispatch costs we use the lowest marginal cost of the piece-wise linear cost curve for each generating unit as an indicative value.

\section{A. The discarding principle}

We begin with establishing the general utility of the discarding principle introduced in (4). To facilitate the clear demonstration of this concept, we shall neglect in this sub-section the potential failure of corrective control. For the same purpose, we also adopt a zero tolerance value for the reliability target of (2) and will thus strictly apply all relevant operational limits with respect to any contingency subset $\mathcal{C}_{c} \subseteq \mathcal{C}$. In order to set a frame of reference, we present in table I a brief overview of the application of the $\mathrm{N}-1$ approach and criterion in the two cases of interest. More specifically, table I] summarizes the results of minimizing the summation of preventive control
TABLE I. COSTS OF THE N-1 APPROACH (\$)

\begin{tabular}{ccc}
\hline & Case A & Case B \\
\hline Total Cost & 5330.78 & 5460.72 \\
Preventive Cost & 5330.73 & 5460.66 \\
$\mathbb{E}\{$ Corrective Cost $\}$ & 0.05 & 0.06 \\
\hline
\end{tabular}

TABLE II. RESIDUAL RISK OF THE N-1 APPROACH (\$)

\begin{tabular}{ccc}
\hline & Case A & Case B \\
\hline (A12-1,A13-1) & 39.85 & 69.45 \\
(A18,A20) & 36.23 & 63.14 \\
(A25-1,A25-2) & 37.13 & 64.72 \\
(A30,A34) & 48.9 & 85.24 \\
Other common mode outages & 0 & 0 \\
\hline Total = Residual Risk & 162.11 & 282.55 \\
\hline
\end{tabular}

costs and probability-weighted corrective control costs, shown in the second and third rows respectively, in anticipation of the possible outage of any single transmission component.

In table II we present the assessment of the residual risk implied by the N-1 approach, which, in these case studies, refers to the possible occurrence of any common mode double outage ${ }^{2}$ Particularly, we list in this table the contribution of each common mode forced outage to the residual risk of the $\mathrm{N}-1$ approach along with the total residual risk value. Although not explicitly shown, we must clarify that, all the contingencies with non-zero values were found in our simulations to lead to the loss of the full system load. Accordingly, we notice that the difference in residual risk between cases $A, B$ can be attributed to several factors. Firstly, the system is slightly more loaded in case $B$, hence more vulnerable to the potential occurrence of any contingency. At the same time, failure probabilities, as well as the severity of a potential service interruption are assumed to be greater in case $B$. Particularly concerning the latter, we must notice that both the voll as well as the consumer demand are greater, leading to a notable increase in the severity of a service interruption.

Exploiting the findings shown in table II] we move on to consider the following numerical values for the accuracy threshold: 1) $\Delta E_{R T}=\$ 165$, i.e. a value slightly above the residual risk implied by the $\mathrm{N}-1$ approach in case $\mathrm{A}$, and 2) $\Delta E_{R T}=\$ 285$, i.e. a value slightly above the residual risk implied by the N-1 approach in case B.

1) $\Delta E_{R T}=\$ 165:$ As shown in table [I] in case $A$ the residual risk of the $\mathrm{N}-1$ approach is marginally smaller than the considered discarding threshold. As per the discarding principle of the probabilistic RMAC, in any such case the

\footnotetext{
${ }^{2}$ We should clarify that corrective control is not taken into account for such an assessment, since as per the N-1 approach the double outages are neglected. Hence there are no pre-computed, contingency specific corrective actions to counteract the possible occurrence of these events.
} 
TABLE III. RMAC CosTs (\$) vs $\Delta E_{R T}$

\begin{tabular}{ccc|cc}
\hline & \multicolumn{2}{c|}{$\Delta E_{R T}=\$ 165$} & \multicolumn{2}{c}{$\Delta E_{R T}=\$ 285$} \\
& Case A & Case B & Case A & Case B \\
\hline Total Cost & 5330.78 & 5988.98 & 5330.78 & 5460.72 \\
Preventive Cost & 5330.73 & 5988.83 & 5330.73 & 5460.66 \\
$\mathbb{E}\{$ Corrective Cost $\}$ & 0.05 & 0.15 & 0.05 & 0.06 \\
Residual Risk & 162.11 & 154.7 & 186.27 & 282.55 \\
\hline
\end{tabular}

subset of single outages can be considered as an acceptable approximation, as in $\mathcal{C}_{c} \equiv \mathcal{C}_{N-1} \subseteq \mathcal{C}$.

On the contrary, in case $B$ the (much larger) residual risk value is well beyond the discarding threshold. As per the discarding principle of the probabilistic RMAC, in any such case the contingency subset of concern should be extended with respect to the $\mathrm{N}-1$ approach, as in $\mathcal{C} \supseteq \mathcal{C}_{c} \supset \mathcal{C}_{N-1}$. Inspecting once again table $[\mathrm{I}$. we notice that extending the subset with any two common mode outages should in principle suffice to render the residual risk acceptable. While we began by considering the inclusion of the contingency contributing the most to the residual risk value, i.e. the common mode outage of $(\mathrm{A} 30, \mathrm{~A} 34)$, we have concluded that the inclusion of this contingency in $\mathcal{C}_{c}$ renders the problem in question infeasible. Consequently, we have identified the pair of common mode outages [(A18,A20);(A25-1,A25-2)] that indeed suffices to imply residual risk below the discarding threshold. The residual risk implied by the inclusion of this contingency pair is presented in the second column of table III along with the optimal preventive and expected corrective costs of reliability management with respect to this extended contingency subset. In comparison with table II] we notice that, in this particular case, the reduction of the residual risk is achievable at a more significant cost increase, because the RMAC prescribes that for these contingencies an acceptable response should be guaranteed (here with probability 1 ).

2) $\Delta E_{R T}=\$ 285$ : Let us now discuss a much larger acceptability threshold value, making the residual risk implied by the $\mathrm{N}-1$ approach marginally acceptable in case $B$, table II

We focus here on case $A$ to notice that the residual risk of the $\mathrm{N}-1$ approach, table $\Pi$, is well below such threshold. As per the discarding principle of the probabilistic RMAC, in any such case, a proper subset of the single outages should actually suffice to ensure the real-time reliability management, as in $\mathcal{C}_{c} \subset \mathcal{C}_{N-1} \subset \mathcal{C}$. To identify such a subset, we have performed the residual risk assessment of the initial system operating state, i.e. under the initial realization of power generation and demand. Accordingly we have identified that, in addition to any common mode double outage, the single outage of (A8) can also be discarded while jointly implying acceptable residual risk. The optimal preventive and expected corrective costs of reliability management with respect to the resulting reduced contingency subset, as well as the implied residual risk are listed in the third column of table IIII We
TABLE IV. RMAC Costs (\$) VS TOLERANCE LEVEL

\begin{tabular}{cccc|c}
\hline$\varepsilon_{R T}$ & $10^{-6}$ & $10^{-5}$ & $10^{-4}$ & Ignored failure \\
\hline Total Cost & 8345.08 & 6759.41 & 4516.74 & 6661.67 \\
Preventive Cost & 8334.55 & 6694.90 & 3490.03 & 5988.83 \\
$\mathbb{E}\{$ Corrective Cost $\}$ & 0 & 0 & 0.19 & 0.15 \\
$\mathbb{E}\{$ Severity $\}$ & 10.53 & 64.51 & 1026.52 & 672.69 \\
Residual Risk & \multicolumn{3}{c}{ All equal to : 154.7} & 154.7 \\
\hline
\end{tabular}

notice that there is no difference in the costs of the optimal preventive/corrective actions with respect to table III. This is due to the fact that the outage of (A8) would be handled by means of corrective control on the phase shifting transformers, which is considered here as a costless action. Rather, the only difference appears in the implied residual risk, which is now increased by the contribution of neglecting outage (A8).

\section{B. Reliability target}

We continue by focusing on the reliability target introduced in (2). For this purpose, we henceforth restrict to considering case $B$ for an accuracy threshold of $\Delta E_{R T}=$ $\$ 165$. As detailed in the previous sub-section, we thus consider an extended contingency subset of 41 events, including any single outage, as well as the common mode outages [(A18,A20);(A25-1,A25-2)].

First, let us analyze the effect of different tolerance level values $\varepsilon_{R T}$. To this end, we will arbitrarily assume that the probability of failure of an elementary corrective control operation, i.e. the corrective control of any single generating unit and/or phase shifting transformer, is equal to $(0.1)$.

Table IV] presents an analysis of the outcome of reliability management as per the probabilistic RMAC for different tolerance level value ${ }^{3}$. The last column of this table corresponds to the case wherein the preventive and corrective controls have been computed while neglecting the potential failure of corrective control and with a value $\varepsilon_{R T}=0$. As can be seen in the second row of this table, smaller values of the tolerance level (i.e. a more risk averse attitude) imply greater costs of preventive control. Conversely, both the expected costs of corrective control as well as the expected severity increase for greater tolerance level values. However, the total costs of the three first columns (i.e. our RMAC explicitly modeling the corrective control failures while computing its decisions) are comparable to the last column.

To analyse these results in more detail, table $\mathrm{V}$ categorizes contingencies $c \in \mathcal{C}_{c}$ into sub-groups of preventively and correctively secured as per the probabilistic RMAC. More specifically, the first row of table $\mathrm{V}$ presents the number of contingencies that would be secured preventively, i.e. by means

\footnotetext{
${ }^{3}$ Noting that in the case under consideration reliability management by means of preventive control only is not feasible, we omit from this analysis the value of $\varepsilon_{R T}=0$, since such a value would exclude the use of corrective control given it's non-zero failure probability.
} 
TABLE V. CONTINGENCY CATEgorizATION

\begin{tabular}{cccc|c}
\hline$\varepsilon_{R T}$ & $10^{-6}$ & $10^{-5}$ & $10^{-4}$ & Ignored failure \\
\hline Preventively Secured & 40 & 39 & 35 & 36 \\
Correctively Secured & 1 & 1 & 4 & 5 \\
Secured & $41 / 41$ & $40 / 41$ & $39 / 41$ & $41 / 41$ \\
\hline
\end{tabular}

TABLE VI. PRoBABILITY OF UNACCEPTABLE STATES

\begin{tabular}{cccc|c}
\hline$\varepsilon_{R T}$ & $10^{-6}$ & $10^{-5}$ & $10^{-4}$ & Ignored failure \\
\hline $\mathbb{P}\left\{\mathbf{x} \notin X_{a}\right\}$ & $8.5 \cdot 10^{-7}$ & $5.2 \cdot 10^{-6}$ & $8.3 \cdot 10^{-5}$ & $5.5 \cdot 10^{-5}$
\end{tabular}

of preventive actions. As the tolerance level increases, the number of preventively secured contingencies only decreases since i) lower probability contingencies may not be secured and lead to unacceptable trajectories, since their probability of realization becomes gradually tolerable, and, ii) more contingencies may be secured by means of corrective actions in spite of the possible corrective control failure. The former is exhibited in the first two columns of table $\mathrm{V}$. More specifically, our analysis indicates that, as the tolerance level increases from $10^{-6}$ to $10^{-5}$, the potential realization of the common mode outage of (A25-1,A25-2), with a probability of $4.38 \cdot 10^{-6}$ becomes tolerable. Notice from table IV that this translates into a decrease in the costs of preventive control and an increase of the expected severity, leading to an overall reduction of the total cost.

As for the impact of the potential failure of corrective control, we point to the second row of $\mathrm{V}$, and particularly the difference between the tolerance levels of $10^{-5}$ to $10^{-4}$. Such a change would allow the use of corrective control to secure three more contingencies, namely the single outages of A8, A20 and A28. The probability of realizing these events and the subsequent failure of the identified corrective control actions is $8.55 \cdot 10^{-7}, 5.56 \cdot 10^{-6}$, and $1.75 \cdot 10^{-5}$ respectively.

Let us also notice the differences with respect to the case wherein the possible corrective control failure is ignored while the value of $\varepsilon_{R T}$ is equal to zero, shown as the last column in tables IV] and VI. Firstly, let us discuss this case in contrast to a solution of similar socio-economic cost, i.e. the result obtained while $\varepsilon_{R T}=10^{-5}$. Notice the difference in the components that build our total cost function. Ignoring the failure of corrective control implies a greater severity level, while the acknowledgment of this possibility and the probabilistic reliability target jointly result in a similar increase of the preventive control cost. In other words, the additional features of the proposed RMAC re-arrange the tradeoff between the costs of security and the consequences of insecurity in the interest of the system end-users. Secondly, let us also identify the difference with respect to a result of similar probability of reaching an unacceptable system state, i.e. the result obtained
TABLE VII. RMAC Costs (\$) vs CORRective FAILURe ProbabiLITy

\begin{tabular}{ccccc}
\hline$\pi_{\text {fail }}$ & 0.10 & 0.30 & 0.40 & 0.50 \\
\hline Total Cost & 4516.74 & 5862.93 & 6578.59 & 6720.49 \\
Preventive Cost & 3490.03 & 4655.25 & 5946.76 & 5946.76 \\
$\mathbb{E}\{$ Corrective Cost $\}$ & 0.19 & 0.10 & 0.05 & 0 \\
$\mathbb{E}\{$ Severity $\}$ & 1026.52 & 1205.58 & 629.78 & 773.73 \\
Residual Risk & & All equal to : 154.7 \\
\hline
\end{tabular}

while $\varepsilon_{R T}=10^{-4}$. We notice that the proposed RMAC can indeed achieve a relatively similar level of reliability at a considerably lower total cost.

Moving on, in table VII we use the tolerance level of $10^{-4}$ and present the adaptability of the reliability target to the probability of failure of any elementary corrective control operation, denoted for simplicity $\pi_{f a i l}$ in this table. Between the values of $[0.1,0.3]$ we can firstly notice that the increase of the failure probability translates into an increase of the expected severity value. Preventive control costs appear to also increase, since the use of corrective control becomes more limited both due to the increased expected severity implied by its potential failure and since the probability of realizing an unacceptable system state becomes larger. Notably, between the values of $[0.3,0.4]$ the expected severity reduces. Our detailed results indicate that this arises from the fact that fewer contingencies would be secured correctively, to avoid the potential realization of corrective control failure. In the last column of table VII we demonstrate that, for the probability value of 0.5 , no contingency would be secured correctively. Indeed, in this case, the probability of failure of any control action including at least two elementary operations would be equal to 1, i.e. corrective control is completely ineffectual in practice. The expected severity level listed in table VII for this case corresponds to the fact that, as per the tolerance level of $10^{-4}$, the low probability outages [(A24);(A18,A20);A(251,A25-2)] would not be secured.

Finally, let us underline that in this particular example the probability of corrective control failure has no impact on the residual risk. Firstly, recall from subsection IV-A that we have not considered any corrective action for contingencies $c \in \mathcal{C} \backslash \mathcal{C}_{c}$. Hence, the increase in corrective control failure probability would not increase residual risk. Secondly, although the increase in the corrective control failure probability drives the decision making towards a more secure preventive dispatch, this does not suffice here to reduce the potential consequences of neglected contingencies $c \in \mathcal{C} \backslash \mathcal{C}_{c}$.

We conclude by establishing the adaptability to the (possibly) different failure probabilities of elementary corrective control operations. For this purpose, we shall assume that the elementary corrective control operation of phase shifting transformers has a failure probability of 0.2 while the failure probability of corrective generation redispatch is re-set to 0.1 . Table VIII lists the differences in the types of corrective 
TABLE VIII. CORRECTIVE CONTROL FAILURE PROBABILITY EFFECT

\begin{tabular}{ccc}
\hline Contingency & $\pi_{\text {fail }}^{\mathrm{PST}}=0.1$ & $\pi_{\text {fail }}^{\mathrm{PST}}=0.2$ \\
\hline A8 & Redispatch \& phase shift & Redispatch only \\
A25-1,A25-2 & Redispatch \& phase shift & Redispatch only \\
\hline
\end{tabular}

control actions identified for this set of probability values, in contrast to the case where the probability of the phase shifting transformer corrective operation (denoted for simplicity $\pi_{\text {fail }}^{\mathrm{PST}}$ in this table) is also equal to 0.1. As shown, when the failure probability of the phase shifting transformers is increased, in the second column, the (in principle) more expensive generation redispatch becomes the preferred corrective action. The resulting difference in the total cost between these two instances was found to be equal to $\$ 111.98$.

\section{CONCLUDING DISCUSSION}

In this last section we discuss the proposed RMAC in the light of several considerations. We start by recapitulating the motivation and rationale behind such an RMAC; then we discuss several possible directions for upgrading the proposal, in particular to take into account practical needs in real-life system operation; finally, we provide a perspective on how to pursue the endeavour to establish a consistent approach to reliability management over the different contexts and horizons of TSOs' tasks.

\section{A. Motivation, rationale, and findings - in hindsight}

Reliability management in real-time operation is mainly a matter of arbitrating in the best way between potentially costly preventive control actions and often cheap (in expectation) but less dependable corrective control actions. In order to carry out this arbitration, we have proposed a probabilistic approach, modelling the probabilities of contingencies and the probabilities of corrective control failure modes, combined with an overall cost function aggregating preventive and corrective control costs with a monetised measure of the impact of service interruptions. In addition to this, which is an approach already discussed in the literature, we have also augmented our RMAC with an explicit reliability target in the form of a chance constraint on the non-acceptable system responses allowing one to force decisions so that they avoid such conditions, and a discarding principle allowing one to consistently judge whether a certain subset of contingencies is sufficiently rich in order to assess and optimise the realtime operation decisions.

Since the proposed RMAC explicitly recognizes the spatiotemportal variation of probabilities and costs, the arbitration between preventive and corrective controls - linked to sets of contingencies to be explicitly covered - is more flexible and can adapt automatically to prevailing conditions. The way this arbitration could work in practice has been shown on the basis of a detailed case study, highlighting the impact of the variability of costs of service interruptions, of contingency probabilities (and beyond the single outages typically covered in $\mathrm{N}-1$ practice), and of corrective control failure probabilities. This preliminary study already reveals a number of interesting findings, particularly the flexibility and adaptability of arbitrating between preventive and corrective controls as per our proposal.

\section{B. Possible upgrades of the proposed real-time RMAC}

In our physical models used to formulate the assessment of severities and the optimisation of real-time decisions in the context of our case study, we have adopted quite simplistic (mostly DC and quasi-static) assumptions, which greatly facilitate the implementation of algorithms and the analysis of results, but which may be considered as oversimplified for reallife practical use on stability or voltage limited systems. The study of the proposed RMAC in the context of less restrictive physical modelling assumptions is of course acknowledged as one of the first next steps to address. In relation to this issue, we refer the interested reader to [4] for a recent critical survey of the state-of-the-art in Security Constrained Optimal Power Flow (SCOPF).

Additionally, we have used a rather simple model to account for corrective control failure probabilities, assuming that it can be computed as a sum of probabilities of individual control action failures, and without having access to realistic data concerning the corresponding orders of magnitude. The validation/refinement of such corrective control failure models is as well of paramount importance for the practical applicability of the proposed approach.

We have completely neglected the possible residual uncertainties about the load and generation over the real-time horizon $\Delta t \sim 15^{\prime}-60^{\prime}$. In some systems, this assumption may be unrealistic, so that the RMAC would need to take as well into account this kind of uncertainty. Obviously, from a pure theoretical point of view, such additional uncertainties could be incorporated in the proposed RMAC, but we believe that one should rather tackle this problem in a broader context where multiple stages of real-time preventive/corrective control decisions would be assessed and optimised over a longer "lookahead" horizon; in principle also covering operation planning decision making. The development of such a look-ahead mode multi-stage model of reliability management is a subject of further research.

Let us finally underline the aspect of multi-TSO coordination for reliability management. While we did not address this issue in the present work, we believe that this question deserves specific additional research in the context of the probabilistic RMAC that is proposed, since its decision making logic may reveal other problems than those currently handled when considering the coordination with the $\mathrm{N}-1$ approach.

\section{Towards the migration from the N-1 approach}

As advocated in this paper, there is a rather clear need to migrate away from the current N-1 strategy of real-time reliability management. Such a migration will however be 
possible only under several conditions, that we briefly discuss below.

1) Coping with data \& algorithmic limitations: The proposed approach suffers from two main practical barriers, namely the need to rely on data not used in the classical N1 approach (severity function, probabilities of contingencies and of corrective control failures) and additional computational requirements.

The additional data items are in principle functions of time and space in a way which is not fully understood as of today. Therefore, any practical application of the proposed approach needs to be preceded by sensitivity studies to assess the impact of such data limitations, and identify the most important factors of the additional modelling requirements.

Be that as it may, we can already recognize that, similarly to current tools for preventive/corrective control optimisation, our approach calls for a SCOPF type of computation (over a subset of covered contingencies) and a contingency response evaluation module (over a typically much larger set of contingencies). With respect to the corresponding modules tailored to the $\mathrm{N}-1$ criterion, both modules are intrinsically more complex, and hence will need further work to ensure sufficient efficiency in the context of large systems of interest and in practical applications. We however don't see any fundamental scalability barrier in this respect.

2) Objectifying the value of probabilistic criteria: To enable the move away from the $\mathrm{N}-1$, we need first to carry out pilot tests (in simulation) where we compare in foresight the interest of using the proposed RMAC rather than the current N-1 approach, while assessing the impact in terms of socio-economic costs and benefits of the different stakeholders; we also need to study the practical useability of the proposed methods, and in particular the interpretability of its recommendations by power system operators.

3) From short-term to long-term decision making: As soon as the usefulness and practicality of the new RMAC is established in the context of real-time operation, we need to see what are the implications of its use in real-time, when it comes to decision making in other - longer-term - decision making contexts, such as operation planning (a few hours, days, weeks ahead in time), asset management (maintenance, inspection, and replacement, over months or many years) [18] and grid development (to be justified over decades). In principle these later decision making contexts aim at choosing their strategies while targeting to ensure the reliable operation of the system in real-time. Hence, they need to cover longer-term uncertainties while using as a reliability target the possible operation of the system under the chosen RMAC. Therefore, any change in real-time operation, as concerns the reliability management approach and criterion, needs to be reflected well in the formulation of the corresponding longer-term reliability assessment and control problems.

All the above considerations are part of those addressed in the FP7 project GARPUR, and we refer the interested reader to the web page http://www.garpur-project.eu to see how work will progress in these lines during the next years.

\section{REFERENCES}

[1] A. Patton, "A probability method for bulk power system security assessment: I-basic concepts," Power Apparatus and Systems, IEEE Transactions on, no. 1, pp. 54-61, 1972.

[2] GARPUR Consortium, "Current practices, drivers and barriers for new reliability standards," 7th framework programme, EU Commission grant agreement 608540, May 2014. [Online]. Available: http://www.garpur-project.eu/deliverables

[3] F. Capitanescu, J. Martinez Ramos, P. Panciatici, D. Kirschen, A. Marano Marcolini, L. Platbrood, and L. Wehenkel, "State-of-theart, challenges, and future trends in security constrained optimal power flow," Electric Power Systems Research, vol. 81, no. 8, pp. 1731-1741, 2011.

[4] F. Capitanescu, "Critical review of recent advances and further developments needed in $\{\mathrm{AC}\}$ optimal power flow," Electric Power Systems Research, vol. 136, pp. 57 - 68, 2016.

[5] GARPUR Consortium, "Functional analysis of system operation processes," 7th framework programme, EU Commission grant agreement 608540, Feb. 2015. [Online]. Available: http://www.garpur-project.eu/ deliverables

[6] E. Karangelos, P. Panciatici, and L. Wehenkel, "Whither probabilistic security management for real-time operation of power systems ?" in Bulk Power System Dynamics and Control (iREP) - IX (iREP), 2013 iREP Symposium, 2013.

[7] K. R. W. Bell, D. Kirschen, R. N. Allan, and P. Kelen, "Efficient monte carlo assessment of the value of security," in 13th Power System Computation Conf., 1999.

[8] M. Rios, D. S. Kirschen, D. Jayaweera, D. P. Nedic, R. N. Allan et al., "Value of security: modeling time-dependent phenomena and weather conditions," Power Systems, IEEE Transactions on, vol. 17, no. 3, pp. 543-548, 2002

[9] C. Grigg, P. Wong, P. Albrecht, R. Allan, M. Bhavaraju, R. Billinton, Q. Chen, C. Fong, S. Haddad, S. Kuruganty, W. Li, R. Mukerji, D. Patton, N. Rau, D. Reppen, A. Schneider, M. Shahidehpour, and C. Singh, "The IEEE Reliability Test System-1996. a report prepared by the reliability test system task force of the application of probability methods subcommittee," vol. 14, no. 3, pp. 1010 -1020, aug 1999.

[10] E. Karangelos and L. Wehenkel, "Probabilistic reliability management approach and criteria for power system real-time operation supplementary material," Oct. 2015. [Online]. Available: http://hdl. handle.net/2268/193403

[11] R. Billinton and G. Singh, "Application of adverse and extreme adverse weather: modelling in transmission and distribution system reliability evaluation," Generation, Transmission and Distribution, IEE Proceedings-, vol. 153, no. 1, pp. 115-120, Jan 2006.

[12] A. J. Wood and B. F. Wollenberg, Power Generation, Operation, and Control, 2nd ed. New York: Wiley Interscience, 1996.

[13] R. Billinton and R. N. Allan, Reliability Evaluation of Power Systems, 2nd ed. New York: Plenum, 1996.

[14] J. Yan, Y. Tang, H. He, and Y. Sun, "Cascading failure analysis with dc power flow model and transient stability analysis," Power Systems, IEEE Transactions on, vol. 30, no. 1, pp. 285-297, Jan 2015.

[15] M. Eppstein and P. Hines, "A "random chemistry" algorithm for identifying collections of multiple contingencies that initiate cascading failure," Power Systems, IEEE Transactions on, vol. 27, no. 3, pp. 1698-1705, Aug 2012.

[16] M. Fotuhi-Firuzabad and R. Billinton, "Impact of load management on composite system reliability evaluation short-term operating benefits," Power Systems, IEEE Transactions on, vol. 15, no. 2, pp. 858-864, May 2000.

[17] F. Bouffard, F. Galiana, and A. Conejo, "Market-clearing with stochastic security-part ii: case studies," Power Systems, IEEE Transactions on vol. 20, no. 4, pp. 1827-1835, Nov 2005.

[18] G. Dalal, E. Gilboa, and S. Mannor, "Distributed scenario-based optimization for asset management in a hierarchical decision making environment," to appear in 19th Power System Computation Conf. (PSCC 2016), 2016. 